\title{
ABSOLUTE MAGNITUDES OF O STARS FROM A STUDY OF VISUAL MULTIPLE SYSTEMS
}

\author{
M. L. BURNICHON
}

Institut d'Astrophysique de Paris, France

\begin{abstract}
From a careful study of physical multiple systems, it has been possible to derive differentially absolute magnitudes and intrinsic colours of $O$ stars from the corresponding quantities, relatively well-known, of companions of these stars.
\end{abstract}

A study of wide physical multiple systems - whose brightest member is an $\mathrm{O}$ star or a blue supergiant, surrounded by less massive main sequence fainter companions of type B or sometimes A - was made in the Paris BCD spectrophotometric system $\left(\lambda_{1}, D, \varphi_{b}, \varphi_{u v}\right.$ parameters) and in the $U B V$ photometric system (Burnichon, 1972).

One aim of this study was to determine the absolute magnitudes of bright components from those, which are much better known, of faint components. We assume that all stars of a same system are similarly reddened by interstellar absorption. This is justified because firstly, the reddening is not large (mean $E(B-V)=0.40$ ) as we chose the less reddened systems. Also most of the absorption is foreground absorption and it is hardly likely that it could be variable because of the small size of the systems studied. Moreover, no abnormal behaviour of reddening law was found spectrophotometrically. Finally, if the reddenings were different, the differences would be very small (some hundredths in $B-V$ ) and the error in magnitude found would only be of the order of 0.1 .

The absolute magnitudes of the faint components were derived from their $\left(\lambda_{1}, D\right)$ spectral classification and from the surface $(\Sigma)$ luminosity calibration (Chalonge and Divan, 1973). The absolute magnitudes of the bright components were derived differentially.

For B supergiants, the results are in very good agreement with the surface $(\Sigma)$ calibration.

For $\mathrm{O}$ stars, we find a very large dispersion (the absolute magnitudes approximately lie between -3.5 and -7 ) difficult to explain by uncertainties of the method used, and which is probably real.

Nevertheless, it must be emphasized that we observed very few systems (14 of which only 7 have a central star of type 0 ), and we need more observations to confirm these results and to search for correlations with the precise O-type classification and with the ' $\mathrm{f}$ ' characteristic.

Note. Another result $I$ would only like to mention briefly concerns the intrinsic colours of $O$ stars. The $(B-V)_{0}$ obtained for these stars lie between -0.29 and -0.25 , that-is-to-say are redder than the classical values generally admitted (the mean color corresponds to the color of B1 main sequence stars).

Perhaps this result could be explained by circumstellar reddening...? 


\section{References}

Burnichon, M. L.: 1972, 'Contribution à l'étude des couleurs intrinsèques et des magnitudes absolues des étoiles bleues de grande luminosité à partir de l'observation de systèmes multiples', Thèse.

Chalonge, D. and Divan, L.: 1973, Astron. Astrophys. 23, 69.

\section{DISCUSSION}

Jaschek: What was the average angular separation between the components of your pairs? Are you sure they are physical and not optical?

Burnichon: $15^{\prime \prime}$ to $60^{\prime \prime}$. They are not optical.

Schmidt-Kaler: If you make a plot (again I did so $11 \mathrm{yr}$ ago) $A_{v}$ vs distance for the late B stars you obtain a certain slope within, say, $500 \mathrm{pc}$. the line goes through zero with my intrinsic colour. If you do the same for the early B's you find the same result. If you plot however, the O-stars in the same way, you find the same slope, but the line does not go through zero. There is a 0.9 residual. absorption, probably of circumstellar origin. This effect is also seen in a paper by K. H. Schmidt 